PROCEEDINGS OF THE

AMERICAN MATHEMATICAL SOCIETY

Volume 125, Number 1, January 1997, Pages 121-127

S 0002-9939(97)03509-0

\title{
EXTENSIONS OF MULTIPLIERS AND DILATIONS OF PROJECTIVE ISOMETRIC REPRESENTATIONS
}

\author{
GERARD J. MURPHY
}

(Communicated by Palle E. T. Jorgensen)

\begin{abstract}
An elementary proof of a multiplier extension theorem of M. Laca and I. Raeburn is presented. A very general dilation theorem is also derived.
\end{abstract}

\section{INTRODUCTION}

The problem of extending a multiplier on a subsemigroup to a multiplier on a group has arisen in a number of contexts in recent years. It arises, for instance, in the context of W. B. Arveson's theory of semigroups of endomorphisms. In [1] Arveson showed that every multiplier on the semigroup $\mathbf{R}^{+}$extends to $\mathbf{R}$. This result was extended to the semigroup $[t, \infty)(t>0)$ by P. R. Chernoff in [2] and $H$. Dinh has shown in [3] that a multiplier on an arbitrary discrete subsemigroup of $\mathbf{R}$ admits an extension to the group it generates. More recently, M. Laca and I. Raeburn [5] have obtained far-reaching generalisations of these results. The method they use involves dilation theory. Their multiplier extension theorem is deduced as a consequence. The dilation theory they derived has been used by the author in [6], where aspects of the theory of crossed products of $C^{*}$-algebras by semigroups of endomorphisms are developed. It seems likely that the Laca-Raeburn dilation theorem will admit other applications.

In this paper we present extension and dilation theorems related to those of [5]. In particular, we give a new proof of the multiplier extension theorem. Although our method is similar to that of [5], our proof is completely elementary and does not require the dilation theorem. In addition, we present a number of other extension theorems.

We also prove a dilation theorem more general than that proved by Laca and Raeburn. Our approach is radically different from that of these authors. Their proofs, although very elegant, are developed along lines rather different from those of classical dilation theory as presented, for example, by D. E. Evans and J. T. Lewis in [4]. The theory in [4] involves positive definite kernels and their Kolmogorov decompositions. The many results that fall within its scope include the unitary dilation results on groups due to M. Naimark and B. Sz.-Nagy and the dilation theorem of W. F. Stinespring on completely positive maps. In this paper we apply this theory to obtain the Laca-Raeburn dilation theorem. As well as having the advantage of placing the theorem in the classical setting and thereby presenting a

Received by the editors February 22, 1995 and, in revised form, May 22, 1995.

1991 Mathematics Subject Classification. Primary 47A20, 43A35, 46L99.

(C)1997 American Mathematical Society 
"natural" proof, our approach also has the advantage that we are able to prove a more general result (Theorem 3.1).

The paper is organised as follows: In $\S 2$ we obtain a number of extension results, including the one on multiplier extensions discussed above. Section 3 is concerned with dilation theory and the principal result is Theorem 3.1. The Laca-Raeburn dilation theorem (Theorem 3.3) is shown to be a consequence of it.

\section{EXTENSION OF MULTIPLIERS}

Throughout the paper the term semigroup will signify a semigroup with unit. A subsemigroup of a semigroup signifies a subset closed under the operation and containing the unit. We shall usually write the operation multiplicatively and denote the unity by $e$.

An involution on a semigroup $S$ is a function, $s \mapsto s^{*}$, from $S$ to itself having the properties that for all $s, t \in S$, we have $(s t)^{*}=t^{*} s^{*}$ and $\left(s^{*}\right)^{*}=s$. Automatically (and trivially), $e^{*}=e$.

We call a pair consisting of a semigroup together with an involution a $*$-semigroup.

2.1. Example. An involution on a group is defined by setting $x^{*}=x^{-1}$.

2.2. Example. Let $A$ be a unital $C^{*}$-algebra. Define an equivalence relation $\sim$ on $A$ by setting $a \sim b$ if there exists a scalar $\lambda$ of unit modulus for which $b=\lambda a$. Let $S$ be the set of equivalence classes and $\pi$ the canonical surjection from $A$ onto $S$. We make $S$ into a semigroup by setting $\pi(a) \pi(b)=\pi(a b)$ and we define an involution on $S$ by setting $(\pi(a))^{*}=\pi\left(a^{*}\right)$. We shall call $S$ the projective semigroup associated to $A$.

If $U(A)$ denotes the group of unitaries of $A$, then $\pi U(A)$ is, of course, a group, the projective unitary group of $A$.

If $S$ is a semigroup and $S^{\prime}$ a $*$-semigroup, an isometric representation of $S$ in $S^{\prime}$ is a unital homomorphism $\varphi$ from $S$ to $S^{\prime}$ such that $\varphi(s)^{*} \varphi(s)=e$ for all $s \in S$.

A subsemigroup $S$ of a group $G$ is normal if $x S x^{-1} \subseteq S$ for all $x \in G$.

2.3. Theorem. Let $S$ be a normal, generating subsemigroup of a group $G$ and let $\varphi$ be an isometric representation of $S$ in a $*$-semigroup $S^{\prime}$. Then there exists a unique extension $\varphi^{\prime}$ of $\varphi$ to $G$ having the following properties:

(1) $\varphi^{\prime}(x s)=\varphi^{\prime}(x) \varphi(s)$ for all $x \in G$ and $s \in S$;

(2) $\varphi^{\prime}\left(x^{-1}\right)=\varphi^{\prime}(x)^{*}$ for all $x \in G$.

Proof. Since $S$ is normal and generates $G$, every element $x$ of $G$ can be written in the form $x=s^{-1} t$, for some elements $s$ and $t$ of $S$. From this, uniqueness of $\varphi^{\prime}$ is trivial. To get existence, set $\varphi^{\prime}(x)=\varphi(s)^{*} \varphi(t)$, if $x=s^{-1} t$, where $s, t \in S$. Of course, we must show that this is well defined. To see this, suppose that we can also write $x=u^{-1} v$, where $u, v \in S$. Then $u t=\left(u s u^{-1}\right) v$ and since $u s u^{-1}$ belongs to $S$ and $\varphi$ is a homomorphism, we have

$$
\varphi(u) \varphi(t)=\varphi\left(u s u^{-1}\right) \varphi(v) .
$$


However, $\varphi\left(u s u^{-1}\right) \varphi(u)=\varphi(u) \varphi(s)$, so $\varphi(u)^{*} \varphi\left(u s u^{-1}\right) \varphi(u)=\varphi(s)$, and therefore, $\varphi(u)^{*} \varphi\left(u s u^{-1}\right)^{*} \varphi(u)=\varphi(s)^{*}$. Hence,

$$
\begin{aligned}
\varphi(s)^{*} \varphi(t) & =\varphi(u)^{*} \varphi\left(u s u^{-1}\right)^{*} \varphi(u) \varphi(t) \\
& =\varphi(u)^{*} \varphi\left(u s u^{-1}\right)^{*} \varphi\left(u s u^{-1}\right) \varphi(v) \quad \text { (by (1)) } \\
& =\varphi(u)^{*} \varphi(v)
\end{aligned}
$$

Thus, $\varphi^{\prime}$ is well defined, as claimed. It follows immediately from the definition of $\varphi^{\prime}$ that conditions (1) and (2) are satisfied.

By taking adjoints in condition (1) of the preceding theorem, one sees that $\varphi^{\prime}\left(s^{-1} x\right)=\varphi(s)^{*} \varphi^{\prime}(x)=\varphi^{\prime}\left(s^{-1}\right) \varphi^{\prime}(x)$ for all $s \in S$ and $x \in G$.

The following simple result is in [5]. We shall use it below.

2.4. Corollary. Let $S$ be a normal, generating subsemigroup of a group $G$ and let $\varphi$ be a homomorphism from $S$ to a group $G^{\prime}$. Then $\varphi$ has a unique extension to a homomorphism from $G$ to $G^{\prime}$.

Proof. We apply the theorem with $S^{\prime}=G^{\prime}$, where $G^{\prime}$ has the involution given by inversion. We claim that the extension $\varphi^{\prime}$ of $\varphi$ that results is a homomorphism and a moment's reflection shows that to prove this we need only show that if $s, t \in S$, then $\varphi^{\prime}\left(s t^{-1}\right)=\varphi(s) \varphi(t)^{-1}$. However, this follows immediately from the observation that $\varphi^{\prime}\left(s t^{-1}\right) \varphi(t)=\varphi^{\prime}\left(s t^{-1} t\right)=\varphi(s)$.

A multiplier on a semigroup $S$ is a function $\sigma$, from the Cartesian product $S \times S$ to the unit circle $\mathbf{T}$ in the plane, such that for all elements $s, t$ and $u$ of $S$ :

(1) $\sigma(e, s)=\sigma(s, e)=1$;

(2) $\sigma(s, t) \sigma(s t, u)=\sigma(s, t u) \sigma(t, u)$.

Let $S$ be a semigroup and $H$ a Hilbert space. A projective isometric representation of $S$ on $H$ is a map, $W: x \mapsto W_{x}$, from $S$ to $B(H)$ having the following properties for all elements $s, t \in S$ :

(1) $W_{s}$ is an isometry and $W_{e}=1$;

(2) $W_{s t}=\sigma(s, t) W_{s} W_{t}$, where $\sigma(s, t)$ is some scalar of unit modulus.

It follows from the equations

$$
W_{(s t) u}=\sigma(s t, u) W_{s t} W_{u}=\sigma(s t, u) \sigma(s, t) W_{s} W_{t} W_{u}
$$

and

$$
W_{s(t u)}=\sigma(s, t u) W_{s} W_{t u}=\sigma(s, t u) \sigma(t, u) W_{s} W_{t} W_{u}
$$

that $\sigma(s, t) \sigma(s t, u)=\sigma(s, t u) \sigma(t, u)$. Also, since

$$
W_{s}=W_{s e}=\sigma(s, e) W_{s} W_{e}=W_{e s}=\sigma(e, s) W_{e} W_{s},
$$

we have $\sigma(e, s)=\sigma(s, e)=1$. Hence, the function, $\sigma:(s, t) \mapsto \sigma(s, t)$, is a multiplier on $S$, said to be the multiplier associated to $W$.

If all the $W_{x}$ are unitaries, we say that $W$ is a projective unitary representation.

It is clear that if $W$ is a projective isometric representation on a group, it is automatically a projective unitary representation. Also, $W_{x}^{*}=\sigma\left(x^{-1}, x\right) W_{x^{-1}}$ for all $x$.

A projective isometric representation or projective unitary representation with $\sigma$ an associated multiplier will sometimes be referred to as an isometric $\sigma$-representation or, respectively, a unitary $\sigma$-representation. 
2.5. Example. Given a multiplier $\sigma$ on a left-cancellative semigroup $S$, a projective isometric representation having $\sigma$ associated to it exists. To see this, set $H=l^{2}(S)$ and denote by $\left(\delta_{s}\right)_{s \in S}$ the canonical orthonormal basis. For each element $s \in S$, let $W_{s}$ be the isometry on $H$ that maps $\delta_{t}$ onto $\overline{\sigma(s, t)} \delta_{s t}$. A simple computation shows that $W_{s t}=\sigma(s, t) W_{s} W_{t}$.

Now suppose that $S$ is a normal subsemigroup of a group $G$. Obviously, $\delta_{s t}$ belongs to the range of $W_{s}$ and, since $\delta_{s t}=\delta_{t\left(t^{-1} s t\right)}$, this vector $\delta_{s t}$ also belongs to the range of $W_{t}$. Therefore, the ranges of $W_{s}$ and $W_{t}$ are not orthogonal.

2.6. Theorem (Laca-Raeburn [5]). Let $S$ be a normal, generating subsemigroup of a group $G$ and let $\sigma$ be a multiplier on $S$. Then $\sigma$ extends to a multiplier on $G$.

Proof. Let $W$ be the projective isometric representation on $H=l^{2}(S)$, having $\sigma$ as associated multiplier, of the preceding example. Let $S^{\prime}$ be the projective semigroup associated to the $C^{*}$-algebra $B(H)$. The map $\varphi$ obtained by composing $W$ with the canonical map from $B(H)$ to $S^{\prime}$ is clearly an isometric representation of $S$ in $S^{\prime}$. It has an extension to $G$ given by Theorem 2.3 and we denote this extension by $\varphi$ also. If $x \in G$, choose $W_{x}$ in the equivalence class $\varphi(x)$; if $s \in S$, we may, and we do, keep $W_{s}$ as before. Since $\varphi\left(x^{-1}\right)=\varphi(x)^{*}$, there is a scalar $\mu(x)$ of unit modulus such that $W_{x^{-1}}=\mu(x) W_{x}^{*}$. If $x=s^{-1} t$, where $s, t \in S$, then $\varphi(x)=\varphi(s)^{*} \varphi(t)$, so $W_{x}=\lambda W_{s}^{*} W_{t}$, for some scalar $\lambda$ of unit modulus. Since the ranges of $W_{s}$ and $W_{t}$ are not orthogonal, $W_{s}^{*} W_{t}$ is non-zero. Hence, $W_{x} \neq 0$. If $t \in S$ and $x \in G$, then $\varphi\left(t^{-1}\right) \varphi(x)=\varphi\left(t^{-1} x\right)$, so there exists a scalar $\sigma^{\prime}\left(t^{-1}, x\right)$ of unit modulus such that

$$
W_{t^{-1} x}=\sigma^{\prime}\left(t^{-1}, x\right) W_{t^{-1}} W_{x} .
$$

We relate $\sigma^{\prime}$ to $\sigma$ as follows: If $s, t \in S$, then $W_{s t}=\sigma(s, t) W_{s} W_{t}$ and taking adjoints gives $\overline{\mu(s t)} W_{t^{-1} s^{-1}}=\overline{\sigma(s, t) \mu(t) \mu(s)} W_{t^{-1}} W_{s^{-1}}$. Comparing with equation (2) (with $x=s^{-1}$ ) and using the fact that $W_{t^{-1} s^{-1}} \neq 0$, we see that

$$
\sigma^{\prime}\left(t^{-1}, s^{-1}\right)=\mu(s t) \overline{\mu(s) \mu(t) \sigma(s, t)} .
$$

Now we define a projective unitary representation $U$ of the semigroup $S^{-1}$ on $K=l^{2}(G)$ : If $s \in S$, the unitary $U_{s^{-1}}$ is defined on the canonical orthonormal basis $\left(e_{x}\right)_{x}$ of $K$ by setting $U_{s^{-1}}\left(e_{x}\right)=\overline{\sigma^{\prime}\left(s^{-1}, x\right)} e_{s^{-1} x}$. We claim that

$$
U_{t^{-1} s^{-1}}=\sigma^{\prime}\left(t^{-1}, s^{-1}\right) U_{t^{-1}} U_{s^{-1}}
$$

and to see this we need only show that for all $x \in G$ we have

$$
\sigma^{\prime}\left(t^{-1}, s^{-1} x\right) \sigma^{\prime}\left(s^{-1}, x\right)=\sigma^{\prime}\left(t^{-1}, s^{-1}\right) \sigma^{\prime}\left(t^{-1} s^{-1}, x\right) .
$$

However, this follows by comparing the two ways of writing $W_{t^{-1}\left(s^{-1} x\right)}=W_{\left(t^{-1} s^{-1}\right) x}$ in terms of $W_{t^{-1}} W_{s^{-1}} W_{x}$ (using equation (2)). The subsemigroup $S^{-1}$ of $G$ is normal and generates $G$, and the map $\psi$ from $S^{-1}$ to the projective unitary group $G^{\prime}$ of $B(K)$ obtained by composing $U$ with the canonical map from $U(B(K))$ to $G^{\prime}$ is a homomorphism. Hence, by Corollary 2.4, $\psi$ extends to a homomorphism from $G$ to $G^{\prime}$, that we shall also denote by $\psi$. For each element $x \in G$, choose a unitary $U_{x}$ on $K$ belonging to the equivalence class $\psi(x)$. In particular, if $s \in S$, choose $U_{s^{-1}}$ as before and choose $U_{s}=\mu(s) U_{s^{-1}}^{*}$. Of course, if $s \in S \cap S^{-1}$, we need to check that these choices are compatible. That they are follows from the easily verified fact that if $s \in S \cap S^{-1}$, then $\mu(s)=\overline{\sigma^{\prime}\left(s^{-1}, s\right)}$.

If $x, y \in G$, then $\psi(x y)=\psi(x) \psi(y)$ and therefore there exists a scalar $\sigma^{\prime \prime}(x, y)$ of unit modulus such that $U_{x y}=\sigma^{\prime \prime}(x, y) U_{x} U_{y}$. Hence, the function, $\sigma^{\prime \prime}:(x, y) \mapsto$ 
$\sigma^{\prime \prime}(x, y)$, is a multiplier on $G$. If $s, t \in S$, then $U_{s t}=\sigma^{\prime \prime}(s, t) U_{s} U_{t}$. We get $\overline{\mu(s t)} U_{t^{-1} s^{-1}}=\overline{\sigma^{\prime \prime}(s, t) \mu(t) \mu(s)} U_{t^{-1}} U_{s^{-1}}$ if we take adjoints; hence, by equation (4), $\sigma^{\prime}\left(t^{-1}, s^{-1}\right)=\mu(s t) \overline{\mu(s) \mu(t) \sigma^{\prime \prime}(s, t)}$ and consequently, by equation $(3), \sigma^{\prime \prime}(s, t)=$ $\sigma(s, t)$. Thus, the multiplier $\sigma^{\prime \prime}$ on $G$ extends $\sigma$.

\section{Dilation THEORY}

Let $X$ be a non-empty set and let $H$ be a Hilbert space. Recall that a map $K$ from $X \times X$ to $B(H)$ is a positive definite kernel if for every positive integer $n$ and elements $x_{1}, \ldots, x_{n}$ of $X$, the operator matrix $\left(K\left(x_{i}, x_{j}\right)\right)$ in the $C^{*}$-algebra $M_{n}(B(H))$ is positive.

If $K$ can be written in the form $K(x, y)=V(x)^{*} V(y)$, where $V$ is a map from $X$ to $B\left(H, H_{V}\right)$, for some Hilbert space $H_{V}$, then $K$ is automatically positive definite. Such a map $V$ is said to be a Kolmogorov decomposition of $K$. Moreover, if, in addition, $H_{V}$ is the closed linear span of the set $\bigcup_{x} V(x) H$, then $V$ is said to be minimal.

The fundamental result in this connection asserts that if $K$ is a positive definite kernel, then it admits a minimal Kolmogorov decomposition; moreover, if $V$ and $V^{\prime}$ are two such decompositions for $K$, then there exists a unique unitary operator $U$ from $H_{V}$ onto $H_{V^{\prime}}$ such that $V^{\prime}(x)=U V(x)$, for all $x \in X$.

For a nice presentation of this theory, see [4].

For the rest of this section, $G$ will denote a group, $\sigma$ a multiplier of $G$ and $S$ a normal, generating subsemigroup of $G$.

Let $H$ be a Hilbert space. We say that a map, $W: x \mapsto W_{x}$, from $G$ to $B(H)$ is $\sigma$-positive definite if the map $K$ on $G \times G$ defined by setting $K(x, y)=$ $\sigma\left(x^{-1}, x\right) \overline{\sigma\left(x^{-1}, y\right)} W_{x^{-1} y}$ is positive definite. We define a (minimal) Kolmogorov decomposition for $W$ to be a (minimal) Kolmogorov decomposition for $K$.

The following result is well known in the case that $\sigma \equiv 1$, see [4, Corollary 2.6].

3.1. Theorem. Let $H$ be a Hilbert space and $W$ a $\sigma$-positive definite map on $G$ with values in $B(H)$. Then there exists a Hilbert space $H^{\prime}$, an operator $T \in$ $B\left(H, H^{\prime}\right)$ and a unitary $\sigma$-representation $U$ of $G$ on $H^{\prime}$ such that $W_{x}=T^{*} U_{x} T$, for all $x \in G$. Moreover, $H^{\prime}$ is the closed linear span of the set $\bigcup_{x} U_{x} T H$.

Proof. Let $V$ be a minimal Kolmogorov decomposition of $W$ and set $H^{\prime}=H_{V}$. Let $x, y, z \in G$. Then it is routine to verify that

$$
\sigma\left(x^{-1}, z^{-1}, z x\right) \sigma(z, x) \sigma\left(x^{-1}, y\right)=\sigma\left(x^{-1}, z^{-1}, z y\right) \sigma(z, y) \sigma\left(x^{-1}, x\right)
$$

and it follows from this that

$$
\begin{aligned}
V(z x)^{*} V(z y) & =\sigma\left(x^{-1} z^{-1}, z x\right) \overline{\sigma\left(x^{-1} z^{-1}, z y\right)} W_{x^{-1} z^{-1} z y} \\
& =\sigma\left(x^{-1}, x\right) \overline{\sigma\left(x^{-1}, y\right) \sigma(z, x)} \sigma(z, y) W_{x^{-1} y} \\
& =\overline{\sigma(z, x)} \sigma(z, y) V(x)^{*} V(y) .
\end{aligned}
$$

Hence, the map, $x \mapsto \overline{\sigma(z, x)} V(z x)$ is another minimal Kolmogorov decomposition for $W$. Consequently, there exists a unique unitary $U_{z} \in B\left(H^{\prime}\right)$ such that $U_{z} V(x)=$ $\overline{\sigma(z, x)} V(z x)$, for all elements $x \in G$. Since we have

$$
\begin{aligned}
U_{y} U_{z} V(x) & =\overline{\sigma(y, z x) \sigma(z, x)} V(y z x) \\
& =\overline{\sigma(y, z) \sigma(y z, x)} V(y z x)=\overline{\sigma(y, z)} U_{y z} V(x),
\end{aligned}
$$


and the set $\bigcup_{x} V(x) H$ has dense linear span in $H^{\prime}$ (by minimality of $V$ ), therefore $U_{y z}=\sigma(y, z) U_{y} U_{z}$. Thus, the map, $U: x \mapsto U_{x}$, is a projective unitary representation of $G$ with $\sigma$ as associated multiplier. To complete the proof, set $T=V(e)$. Then $T^{*} U_{x} T=\overline{\sigma(x, e)} V(e)^{*} V(x e)=V(e)^{*} V(x)=W_{x}$. Also, $U_{x} T H=V(x) H$ and therefore $H^{\prime}$ is the closed linear span of the set $\bigcup_{x} U_{x} T H$.

The projective representation $U$ is called a dilation of $W$. If $W$ is unital, that is, $W_{e}=1$, then $T$ is an isometry, since $T^{*} T=T^{*} U_{e} T=W_{e}=1$.

3.2. Theorem. Let $H$ be a Hilbert space and let $W: S \rightarrow B(H)$ be a projective isometric representation with associated multiplier the restriction of $\sigma$ to $S$. Then there exists a unique extension $W^{\prime}$ of $W$ to $G$ having the following properties:

(1) $W_{x s}^{\prime}=\sigma(x, s) W_{x}^{\prime} W_{s}$ for all $x \in G$ and $s \in S$;

(2) $W_{x}^{\prime *}=\sigma\left(x^{-1}, x\right) W_{x^{-1}}^{\prime}$ for all $x \in G$.

Moreover, $W^{\prime}$ is $\sigma$-positive definite.

Proof. Uniqueness of $W^{\prime}$ is obvious. To see existence, suppose that $x=s^{-1} t$, where $s, t \in S$, and set $W_{x}^{\prime}=\sigma\left(s^{-1}, t\right) \overline{\sigma\left(s^{-1}, s\right)} W_{s}^{*} W_{t}$. Of course, it must be checked that this is well defined; the proof that this is so-which we omit - goes along the same lines as the proof of Theorem 2.3, with the extra requirement that the scalar factors involving $\sigma$ have to be kept track of. Once it is seen that $W^{\prime}$ is well defined, conditions (1) and (2) follow by routine computations. It remains to show that $W^{\prime}$ is $\sigma$-positive definite. Thus, if $x_{1}, \ldots, x_{n}$ belong to $G$, we must show positivity of the operator matrix $\left(V_{i j}\right)$, where

$$
V_{i j}=\sigma\left(x_{i}^{-1}, x_{i}\right) \overline{\sigma\left(x_{i}^{-1}, x_{j}\right)} W_{x_{i}^{-1} x_{j}}^{\prime} .
$$

We claim that there exists an element $s \in S$ such that $s x_{1}, \ldots, s x_{n} \in S$. To see this, write $x_{i}=v_{i} u_{i}^{-1}$, where $u_{i}, v_{i} \in S$. Then, for $s=u_{1} \cdots u_{n}$, we have $s x_{i}=u_{1} \cdots u_{i}\left(u_{i+1} \cdots u_{n} v_{i}\right) u_{i}^{-1}$, so $s x_{i} \in S$, as required.

Consequently, for some elements $s, t_{1}, \ldots, t_{n}$ in $S$ we have $x_{i}=s^{-1} t_{i}$; hence, since $\sigma\left(t_{i}^{-1} s, s^{-1} t_{j}\right)=\overline{\sigma\left(t_{i}^{-1}, s\right)} \sigma\left(t_{i}^{-1}, t_{j}\right) \sigma\left(s, s^{-1} t_{j}\right)$, we have

$$
\begin{aligned}
V_{i j} & =\sigma\left(t_{i}^{-1} s, s^{-1} t_{i}\right) \overline{\sigma\left(t_{i}^{-1} s, s^{-1} t_{j}\right)} W_{t_{i}^{-1} t_{j}}^{\prime} \\
& =\sigma\left(t_{i}^{-1} s, s^{-1} t_{i}\right) \overline{\sigma\left(t_{i}^{-1} s, s^{-1} t_{j}\right)} \sigma\left(t_{i}^{-1}, t_{j}\right) \overline{\sigma\left(t_{i}^{-1}, t_{i}\right)} W_{t_{i}}^{*} W_{t_{j}} \\
& =\sigma\left(s, s^{-1} t_{i}\right) \overline{\sigma\left(s, s^{-1} t_{j}\right)} W_{t_{i}}^{*} W_{t_{j}} .
\end{aligned}
$$

Thus, $V_{i j}=V_{i}^{*} V_{j}$, where $V_{i}=\overline{\sigma\left(s, s^{-1} t_{i}\right)} W_{t_{i}}$. Hence, $\left(V_{i j}\right)$ is positive, as required.

3.3. Theorem (Laca-Raeburn [5]). Let $H$ be a Hilbert space and $W: S \rightarrow B(H)$ a projective isometric representation with associated multiplier the restriction of $\sigma$ to $S$. Then there exists a Hilbert space $H^{\prime}$, an isometry $T: H \rightarrow H^{\prime}$, and a unitary $\sigma$-representation $U: G \rightarrow B\left(H^{\prime}\right)$ such that $T^{*} U_{s} T=W_{s}$, for all $s \in S$. Moreover, $H^{\prime}$ is the closed linear span of the set $\bigcup_{x \in G} U_{x} T(H)$.

Proof. This is obtained by applying Theorem 3.1 to the $\sigma$-positive definite map $W^{\prime}$ extending $W$ that is given by Theorem 3.2 . 


\section{REFERENCES}

[1] W. B. Arveson, An addition formula for the index of semigroups of endomorphisms of $B(H)$, Pacific J. Math. 137 (1989), 19-36. MR 90c:47074

[2] P. R. Chernoff, Extensions and triviality of multipliers on subsemigroups of the reals, Semigroup Forum 41 (1990), 237-244. MR 91c:22008

[3] H. Dinh, Multipliers on subsemigroups of the real line, Proc. Amer. Math. Soc. 117 (1993), 783-788. MR 93d:46111

[4] D. E. Evans and J. T. Lewis, Dilations of Irreversible Evolutions in Algebraic Quantum Theory, Comm. Dublin Inst. Adv. Studies Ser. A No. 24 (1977). MR 58:8915

[5] M. Laca and I. Raeburn, Extending multipliers from semigroups, Proc. Amer. Math. Soc. 123 (1995), 355-362. MR 95c:20101

[6] G. J. Murphy, Crossed products of $C^{*}$-algebras by endomorphisms, Integral Equations Operator Theory 24 (1996), 298-319. CMP 96:08

Department of Mathematics, University College, Cork, Ireland

E-mail address: gerard.murphy@ucc.ie 